It seems that there is still considerable uncertainty about the interpretation of upper atmosphere wind data, although most authors now accept that internal atmospheric gravity waves play an important part at heights between 80 and 150 kilometres. Before a better understanding can be obtained of the dynamies of this very important region of the atmosphere, further observational data are required. As noted by Bedinger et al., existing techniques could be used to make measurements throughout the night and also to investigate horizontal variations in the vertical profile using a series of observing sites. The measurement of daytime winds above the meteor region requires the development of new techniques. Analysis of satellite orbits indicates that the atmosphere is, on the average, rotating faster than the Earth at heights above about $200 \mathrm{~km}$ (King-Hele and Allan, Space Sci. Revs., 6, $248 ; 1966)$. This technique has not revealed fine structure, and the average speed is usually weighted towards daytime conditions. In view of the high rotational speeds obtained, however, direct experimental evidence of the winds is highly desirable; this would also require the development of new techniques.

\section{Solid-Solid Interfaces}

\section{from a Correspondent}

THere are numerous phenomena in ceramic and metallurgical studies which can be traced back either to the intrinsic nature of interfaces (for example, the cohesive strength) or to secondary effects such as the equilibrium segregation of other species. These phenomena formed the framework of a conference held on April 2 and 3 at the National Physical Laboratory and sponsored jointly by the laboratory and the Materials Testing Group of the Institute of Physics. The meeting was attended by about 150 participants of whom about 30 came from other European countries. Several invited review papers were presented, with emphasis on the structural, kinetic, energetic and applied aspects of solid-solid interfaces.

Direct visual evidence from field ion microscopy now confirms that the width of a grain boundary in metals is about one to two atoms. D. McLean (NPL) emphasized the important part that imperfections like kinks and dislocations in grain boundaries may play in phenomena such as the initiation of creep cavities and grain boundary sliding. Electron microscopy shows that, in both iron and nickel, cold working increases the number of grain boundary dislocations, and discussion centred on how the multiplication of grain boundary dislocations occurred.

R. B. Nicholson (University of Manchester) spoke on the nature of precipitate-matrix interfaces and showed that coherency was related to the type and distribution of interfacial dislocations. Elegant transmission electron microscopy by G. Weatherley demonstrated how coherency could be lost by a variety of processes, such as the attraction of dislocations of suitable Burgers vectors from the matrix, these dislocations having the role of accommodating the misfit at the interface.

Solute segregation at interfaces was referred to by a number of speakers. E. D. Hondros (NPL) proposed, on the basis of a Gibbs adsorption analysis of the fall in grain boundary energy with solute content, that the equilibrium segregation in a number of binary systems could be accounted for by levels of enrichment of the order of a monolayer at the grain boundary. By measuring the work of adhesion of metal sessile drops on ceramic substrates, M. Nicholas (AERE, Harwell) showed that minor additions of reactive elements such as titanium and zirconium had a profound strengthening effect. Electron microprobe analysis showed that the segregated zone extended up to seventy microns across the interface, which indicates a different type of segregation. Similarly, Haworth (University of Sheffield) not only proved beyond doubt that the microhardness in the vicinity of grain boundaries in dilute alloys was increased, but also that the width of the affected zone was of the order of microns. The severity of this effect depended on the heat treatment and could be best observed at indentation loads of the order of one gram. It was pointed out in the discussion that a possible mechanism for this effect was the blocking of dislocation sources at grain boundaries by segregated solute atoms.

Theoretical difficulties connected with diffusion down grain boundaries were analysed by G. M. Leak (University of Manchester), who also suggested that observed high temperature internal friction peaks might be associated with grain boundary sliding on a large scale.

A considerable interest in solid-solid interfaces is connected with the possibility of their being preferred paths for the propagation of fracture. D. Hull (University of Liverpool) noted, however, that this is so only in 2 number of well-established cases. He emphasized that a large number of interrelated factors was involved in the failure at interfaces in metals and it was not simply a matter of surface energies. In particular, he noted the possible importance of the ratio between the maximum theoretical tensile strength and the theoretical shear stress.

The conference concluded on a practical note with a paper by P. Bartle (Welding Institute, Cambridge) on diffusion bonding of solids, a process in which the joint is made under pressure at high temperature and in which the condition of the mating surfaces is very important. Very strong joints can be produced in this manner and it is regrettable that the process, first discovered in the Bronze Age, has never been commercially exploited outside the United States and the Soviet Union.

\section{Symposium on Tribology from a Correspondent}

THE first Tribology Liaison Meeting proposed by the Ministry of Technology Committee on Tribology was held in the Institute of Tribology at the University of Leeds on Thursday, April 4. About 140 delegates from local industry and academic institutions attended. The mecting was jointly sponsored by the Ministry of Technology, the Institute of Tribology at the University of Leeds, and the North Midlands branch of the Institution of Mechanical Enginecrs.

$\mathrm{Mr}$ H. P. Jost provided the background to the word and subject of "tribology" in his opening lecture. $\mathrm{He}$ explained the great economic importance of the subject and the neglect it has suffered in education, research and industry in the past. The word tribology, which has introduced an essential unity in the fields of lubrication, friction and wear, was introduced in a Department of Education and Science report 